\title{
A Molecular Assay Allows the Simultaneous Detection of 12 Fungi Causing Fruit Rot in Cranberry
}

\author{
Matteo Conti ${ }^{1}$, Benjamin Cinget $^{1}$, Julien Vivancos ${ }^{2}$, Peter Oudemans ${ }^{3}$, Richard R. Bélanger ${ }^{1 \dagger}$ \\ ${ }^{1}$ Centre de Recherche en Innovation des Végétaux, Université Laval, Quebec City, Québec, Canada \\ ${ }^{2}$ Laboratoire d'expertise et de Diagnostic en Phytoprotection, Quebec City, Québec, Canada \\ ${ }^{3}$ Philip E. Marucci Center for Blueberry and Cranberry Research and Extension, The State University of New Jersey, Chatsworth, \\ NJ, U.S.A.
}

\begin{abstract}
Cranberry fruit rot (CFR) is arguably one of the most limiting factors of cranberry (Vaccinium macrocarpon) production throughout its growing areas. The disease is caused by a group of closely related fungi that require identification using long and cumbersome steps of isolation and microscopic observations of structural features. The objective of this study was to develop a molecular assay to simultaneously detect and discriminate 12 of the most important fungal species reported to be pathogenic on cranberry fruit to facilitate the diagnosis of CFR. As the first approach, internal transcribed spacers and large subunit regions of all fungi were sequenced and confirmed with sequences available in the NCBI database. These data were used to develop primers able to differentiate

differentiated on the basis of a more discriminant marker, the translation elongation factor $1-\alpha$. Two PCR reactions were optimized to clearly delineate the 12 species. The multiplex test was first validated using pure fungal cultures; it was subsequently validated using fruit collected in cranberry beds in eastern Canada. In the latter case, the test was rigorous enough to clearly discriminate the fungal pathogens from contaminants. Within the tested samples, Physalospora vaccinii and Coleophoma empetri were most commonly found. This molecular test offers scientists, diagnosticians, and growers a powerful tool that can rapidly and precisely identify fungi causing CFR so they can implement appropriate control methods.
\end{abstract} seven of the 12 species. The five remaining species, including three in the Phacidiaceae family and two in the Glomerellaceae family, were
Keywords: fruit, multiplex PCR, pathogen detection
Vaccinium macrocarpon (Ait.), the large cranberry, is mainly cultivated in the eastern part of North America; however, it can also be found in the western province of British Columbia and Washington and Oregon in the northwestern United States (Mukezangango 2017). In Canada, $>7000$ ha of cranberry were cultivated in 2016 , generating an economical value exceeding US\$100 million, or $13 \%$ of the fruit farm gate value in the country (Gallardo et al. 2018; Mukezangango 2017). Furthermore, with its nearly 160,000 tons, Canada accounts for $25 \%$ of the entire cranberry production worldwide (Mukezangango 2017).

Among the factors limiting cranberry yield, fungal pathogens represent a major concern and, depending on the regions, can cause losses attributed to cranberry fruit rot (CFR) of $>33 \%$ of total production (Gallardo et al. 2018; Mukezangango 2017; Oudemans et al. 1998; Polashock et al. 2009, 2017). Currently, CFR is considered a disease complex that regroups different fungal pathogen species, often causing similar symptoms (Olatinwo et al. 2003; Oudemans et al. 1998; Polashock et al. 2009, 2017). Recent studies have shown that there are five main fungal species responsible for CFR: Colletotrichum fioriniae (Marcelino \& Gouli ex R.G. Shivas \& Y.P. Tan; teleomorph Glomerella acutata var. fiorinae), Colletotrichum fructivorum (V.P. Doyle, P.V. Oudem. \& S.A. Rehner; teleomorph Glomerella cingulata), Coleophoma empetri Rostr., Phyllosticta vaccinii (Earle), and

${ }^{\dagger}$ Corresponding author: R. Bélanger; richard.belanger@fsaa.ulaval.ca

Funding: This research was supported by grant CRDPJ 508525-17 of the National Sciences and Engineering Council of Canada (to R.R. Bélanger) in collaboration with Ocean Spray Inc., L\&S Cranberry, Groupe Nadeau cranberry, Bieler cranberry, and Bezanson and Chase Cranberry. This work was further supported by grants from the Canada Research Chairs Program (to R.R. Bélanger).

The author(s) declare no conflict of interest.

Accepted for publication 22 May 2019.

(C) 2019 The American Phytopathological Society
Physalospora vaccinii Shear (Arx \& E. Müll) (Damm et al. 2012; Polashock et al. 2009; Weir et al. 2012). However, historically, seven other fungi have been reported to induce CFR: Allantophomoposis lycopodina (Höhn) Carris, Allantophomopsis cytisporea (Fr.) Petr., Phyllosticta elongata Weid. (teleomorph Botryosphaeria vaccinii), Fusicoccum putrefaciens Shear (teleomorph Godronia cassandrae), Monilinia oxycocci (Woronin) Honey, Phomopsis vaccinii Shear (teleomorph Diaporthe vaccinii), and Strasseria geniculata (Berk. \& Broome) Höhn (a species that includes four Strasseria species, like the previously documented $S$. oxycocci [Shear] Shear in cranberry) (McManus et al. 2003; Oudemans et al. 1998; Raj 1983). Because the biology and ecology of the different pathogens involved in CFR are variable (Polashock et al. 2009, 2017), a precise diagnosis will provide necessary information for better disease management and selection of appropriate fungicides targeting the specific fungi.

The diagnosis of CFR is currently based on standard methods applied during the late phases of the disease, when fruit are in an advanced state of decay (Polashock et al. 2017; Wells and McManus 2013). This entails isolating the fungus in pure culture from rotten cranberry fruit and identification based on morphological characteristics (Wells and McManus 2013). However, closely related fungi can be easily confused and species characterization is hampered by the simultaneous presence of several species in a given sample (Weidemann et al. in Polashock et al. 2017). A method allowing the early identification of decay fungi would be helpful for the timely detection of CFR and to establish a preventive management plan.

In recent years, molecular techniques have become a useful ally in many scientific fields, and especially for the diagnosis of plant diseases (Burbank and Ortega 2018; Kostov et al. 2016; Michalecka et al. 2016; Reich et al. 2016; Silva et al. 2017). In particular, (PCR-based methods are used to detect plant pathogens directly from diseased tissues, avoiding the time-consuming isolation and culturing steps (Aslam et al. 2017). Next-generation sequencing (NGS) has considerably reduced both the cost and time of genome sequencing and, in turn, has increased the genetic resources facilitating the design of specific molecular primers (Lang et al. 2010). Many genomic regions have proven valuable for fungal identification at different taxonomic levels (Marin-Felix et al. 2017, 2019). Internal 
transcribed spacers (ITS; ITS1 and ITS2) and structural ribosomal genes, such as the nuclear ribosomal large subunit (LSU) gene, are useful targets for identifying fungi at species levels (Bruns and Shefferson 2004). However, when these regions have low resolution for species discrimination (Schoch et al. 2012), other genomic markers, such as translation elongation factor $1 \alpha$ (TEF1- $\alpha$ ), can be used (MarinFelix et al. 2019; Raja et al. 2017). Simultaneous PCR amplifications with different specific primer pairs in a multiplex PCR assay provide great advantages in the detection of several plant pathogen species, thus saving time and effort (Martinelli et al. 2015). A method of diagnosing CFR was developed previously using reverse dot blot hybridization (Robideau et al. 2008). Multiplex PCR represents a more broadly applicable technique because it does not require manufacturing of macroarrays specific for the assay. Considering the effort and expertise currently required to diagnose the fungi involved in CFR, the development of a standard multiplex PCR-based diagnostic tool would certainly streamline the diagnosis by allowing the identification of the fungal pathogens present in commercial cranberry beds.

The main goal of this study was to develop a multiplex PCR technique to identify 12 of the most important fungal species causing CFR. The specific objectives were to design species-specific primers for each fungus, to develop and optimize a multiplex PCR assay by combining the specific primers for the simultaneous identification of the 12 species, and to validate the reliability of this diagnostic tool by evaluating its sensitivity and specificity for cranberry fruit with CFR symptoms.

\section{Materials and Methods}

Species-specific primers development. Fungal origin and culture. The design and initial validation of the species-specific diagnostic PCR assays were based on pure cultures of 12 fungal species. The species were selected based on their roles in CFR in North America (Olatinwo et al. 2003; Oudemans et al. 1998; Polashock et al. 2009, 2017) and are listed in Table 1 . All 12 fungal strains used in this study were tested for their pathogenicity in cranberry fruit and were found to be able to infect healthy fruit. All fungi were grown on potato dextrose agar (PDA) (Becton Dickinson and Co., Sparks,
MD) medium ( $39 \mathrm{~g} /$ liter), and cultures were kept for 7 days at room temperature $\left(24^{\circ} \mathrm{C}\right)$ before being stored at $4^{\circ} \mathrm{C}$. The cultures were transferred monthly onto fresh medium. For DNA extraction, all fungi were subcultured on PDA with a thin film of cellophane between the medium and the fungal plugs. The fungi were kept at room temperature for 2 weeks, collected in a polypropylene test tube, and then stored at $-80^{\circ} \mathrm{C}$ for $4 \mathrm{~h}$ before lyophilization.

DNA extraction, PCR, and sequencing conditions. Genomic DNA was extracted from lyophilized mycelium grown on a thin film of cellophane over PDA using the E.Z.N.A. plant DNA kit (Omega Biotek, Norcross, GA) according to the manufacturer's instructions for lyophilized samples. Lyophilization was performed at $7.7 \mathrm{~kg} / \mathrm{m} \cdot \mathrm{s}$ using a Labconco Freezone 6 with bulk tray dryer (Labconco Corporation, Kansas City, MO). Then, $50 \mathrm{mg}$ of lyophilized fungal material was placed in a 2-ml screw-cap tube with two 2.8-mm-diameter ceramic beads and ground twice for $45 \mathrm{~s}$ at $6.95 \mathrm{~m} / \mathrm{s}$ each with a $30-\mathrm{s}$ pause between being ground with an Omni Bead Ruptor 24 (Omni International Inc., Kennesaw, GA). Once the extraction protocol was completed, the purity and concentration of nucleic acids were measured with a NanoVue spectrophotometer from GE Healthcare (Chicago, IL), and the eluted DNA was stored at $-20^{\circ} \mathrm{C}$. Amplification of ribosomal DNA including the ITS1, ITS2, 5.8 S rDNA gene, and a part of the $5^{\prime}$ end of the nuclear LSU was performed using universal fungal-specific primers ITS 5 (White et al. 1990) and LR5 (Vilgalys and Hester 1990).

After a primary examination, the three species belonging to the Phacidiaceae family (A. cytisporea, A. lycopodina, and S. geniculata) lacked suitable polymorphisms to create a specific primer design in the ITS and LSU regions. Consequently, TEF1- $\alpha$ was selected for better discrimination of the three species (Marin-Felix et al. 2019). TEF1- $\alpha$ was further amplified using primers EF1728F (Carbone and Kohn 1999) and EF-2 (O’Donnell et al. 1998). The other species were easily resolved. The list of universal primers is shown in Table 2 .

PCR reactions were performed in $25 \mu l$ of Taq DNA polymerase with the ThermoPol Buffer kit (New England Biolabs Inc., Ipswich,

Table 1. List and origin of the 12 fungal species used in this study

\begin{tabular}{|c|c|c|c|c|}
\hline Family & Genus & Species & Supplier & Accession number \\
\hline Phacidiaceae & Allantophomopsis & cytisporea & $\mathrm{ATCC}^{\mathrm{v}}$ & 66955 \\
\hline Phacidiaceae & Allantophomopsis & lycopodina & $\mathrm{ATCC}^{\mathrm{v}}$ & 66958 \\
\hline Botryosphaeriaceae & Phyllosticta $^{\mathrm{w}}$ & elongata & $\mathrm{AAFC}^{\mathrm{x}}$ & C229438 \\
\hline Helotiales incertae sedis & Coleophoma & empetri & Rutgers $^{\mathrm{y}}$ & CE 14-2 \\
\hline Glomerellaceae & Colletotrichum & acutatum & $\mathrm{AAFC}^{\mathrm{x}}$ & $\mathrm{C} 214992$ \\
\hline Glomerellaceae & Colletotrichum & gloeosporioides & $\mathrm{AAFC}^{\mathrm{x}}$ & $\mathrm{C} 212589$ \\
\hline Helotiaceae & Fusicoccum $^{\mathrm{z}}$ & putrefaciens & $\mathrm{AAFC}^{\mathrm{x}}$ & $\mathrm{C} 216021$ \\
\hline Sclerotiniaceae & Monilinia & oxycocci & $\mathrm{ATCC}^{\mathrm{v}}$ & 58544 \\
\hline Diaporthaceae & Phomopsis & vaccinii & $\mathrm{AAFC}^{\mathrm{x}}$ & $\mathrm{C} 233263$ \\
\hline Phyllostictaceae & Phyllosticta & vaccinii & $\mathrm{ATCC}^{\mathrm{v}}$ & 46255 \\
\hline Hyponectriaceae & Physalospora & vaccinii & $\mathrm{AAFC}^{\mathrm{x}}$ & $\mathrm{C} 229655$ \\
\hline Phacidiaceae & Strasseria & geniculata & $\mathrm{AAFC}^{\mathrm{x}}$ & $\mathrm{C} 174523$ \\
\hline
\end{tabular}

v ATCC = American Type Culture Collection (Manassas, VA, U.S.A.).

w Supplied as Botryosphaeria vaccinii.

$\mathrm{x}$ AAFC $=$ Agriculture and Agri-Food Canada (Ottawa, ON, CA).

y Rutgers = Philip E. Marucci Center for Blueberry and Cranberry Research and Extension (Chatsworth, NJ, U.S.A.).

z Supplied as Godronia cassandrae.

Table 2. Names, sequences, and references of the universal primers used in this study to amplify fungal species associated with the fruit rot complex affecting cranberry in Eastern Canada

\begin{tabular}{ll}
\hline Name & \multicolumn{1}{c}{ Primer sequence 5' -3' } \\
\hline $\begin{array}{l}\text { ITS primer } \\
\text { ITS5 }\end{array}$ & GGAAGTAAAAGTCGTAACAAGG \\
LSU primer & TCCTGAGGGAAACTTCG \\
LR5 & \\
$\begin{array}{ll}\text { TEF-1 } \alpha \text { primers } \\
\text { EF1-728F }\end{array}$ & CATCGAGAAGTTCGAGAAGG \\
EF2 & GGA(G/A)GTACCAGT(G/C)ATCATGTT \\
\hline
\end{tabular}


MA) and $10 \mathrm{ng}$ of genomic DNA following the manufacturer's instructions. A T100 Thermal Cycler (Bio-Rad, Hercules, CA) was used for amplifications and programmed with an initial denaturation step at $95^{\circ} \mathrm{C}$ for $30 \mathrm{~s}$, followed by 30 cycles (denaturation at $95^{\circ} \mathrm{C}$ for $30 \mathrm{~s}$; annealing at $45^{\circ} \mathrm{C}$ for $30 \mathrm{~s}$, and elongation at $68^{\circ} \mathrm{C}$ for $2 \mathrm{~min}$ ) and final elongation at $68^{\circ} \mathrm{C}$ for $5 \mathrm{~min}$. Before sequencing, the quantity and quality of PCR products were analyzed using the QIAxcel Advanced system (Qiagen, Hilden, Germany) with the QIAxcel DNA High Resolution Kit and the OM500 program set to the manufacturer's default parameters. PCR products were sequenced at the Institut de Biologie Intégrative et des Systèmes (Quebec City, Québec, Canada) using the Sanger method and analyzed on an automated DNA sequencer 3730xl DNA Analyzer (Thermofisher Scientific, Waltham, MA).

Sequence alignments and specific primer design. All sequences were subjected to a quality check including a manual trim. Then, assembly of the two strands into contiguous sequences (contigs) was performed. To increase the sample size, the sequence of each contig was used in the Basic Local Alignment Search Tool for nucleotides (BLASTn) (Altschul 1997) in the NCBI GenBank database for each of the 12 target species. All sequences found for a given species were aligned, and a consensus sequence was obtained. The 12 consensus sequences were then aligned to identify discriminating regions among the 12 species. Those regions were then tested against the previous alignment to ensure the regions identified were characteristic of the species and not just for a single isolate. All quality checks, alignments, and consensus construction were performed using CLC Genomic Workbench 10 software v.10.0.1 (Qiagen).

Primers were then designed for each species using Primer3Plus software (https://primer3plus.com/cgi-bin/dev/primer3plus.cgi). The following criteria were used for primer design: similar melting temperature (55 to $\left.65^{\circ} \mathrm{C}\right)$ and high GC base pair (bp) content (50 to $60 \%$ ); no self or primer-pair complementation; no complementation with primer pairs of other species; and amplicons of different lengths for unambiguous scoring. Potential matches of designed primers with nontarget species were tested using Primer BLAST tools and restricted to the families of each target species (https:// www.ncbi.nlm.nih.gov/tools/primer-blast/).

Specific primer testing and multiplex development. The specificity and efficiency for each primer pair were evaluated using individual PCR reactions (uniplex reactions). The strict specificity of the primers during uniplex PCR was assessed using mixed fungal DNA templates; the template volume was maintained at $4 \mu \mathrm{l}$, but more than one species was introduced in the reaction. To increase the efficiency of the primer pairs, the optimal annealing temperature and the optimal primer concentration were determined using a thermal gradient $\left(50\right.$ to $\left.65^{\circ} \mathrm{C}\right)$ and a primer concentration gradient $(0.001$ to $0.2 \mu \mathrm{M})$ during PCR cycling. The concentration gradients were processed with $1 / 10$ serial dilutions. These tests were performed using $0.025 \mathrm{U} / \mu \mathrm{l}$ of OneTaq DNA polymerase (New England Biolabs Inc., Ipswich, MA) in a 25- $\mu$ l reaction volume. Amplifications were conducted following the manufacturer's protocol; the parameters optimized for each primer pair, which were selected in accordance with the results of the aforementioned gradients, were considered.

Development of the multiplex PCR assay was achieved by first testing different combinations of the primer pairs with similar annealing temperatures. Testing for efficiency and specificity of the different primer-pair combinations was performed using each individual fungal DNA template against a mixture of multiple primers to establish whether there was competition or interference among primers. Based on the results of uniplex testing, the multiplex PCR assay was optimized by adjusting the primer, salt concentrations, and PCR cycling (annealing temperature and time and the elongation time). During this optimization step, special attention was applied to avoid undesired and multiproduct amplifications and to limit primer competition and dimer formation. New primers were designed if no optimization was possible. Uniplex and multiplex products were visualized using a QIAxcel advanced system (Qiagen), a QIAxcel DNA high-resolution kit, and the OM500 manufacturer's program with the default parameters.
Evaluation of the minimal detection limit for the multiplex assay. As an evaluation of primer-pair sensitivity in the multiplex assay, the minimal detection limit of CFR fungi was determined with serial dilution of pure fungal DNA (from $0.25 \mathrm{ng} / \mu \mathrm{l}$ to $0.25 \times 10^{-3} \mathrm{pg} / \mu \mathrm{l}$ ). For each dilution, a multiplex PCR was performed in a reaction volume of $25 \mu$ under the optimal conditions established previously and with $4 \mu \mathrm{l}$ of DNA template. The presence and intensity of amplicons were controlled using the QIAxcel advanced system (Qiagen).

Validation of multiplex PCR on fruit samples. Plant material. The sensitivity and reliability of the multiplex assay was validated using 117 healthy or infected field-grown cranberry fruit harvested during Fall 2017 on cultivars Stevens, Benlear, or Bergman. These fruit (provided by Jean Pierre Deland, Ocean Spray, Inc., Canada), were randomly harvested from the following four commercial farms: Atocas Somerset (Victoriaville, Quebec City); Les Canneberges de Lourdes (Notre-Dame-de-Lourdes, Quebec City); Canneberges Ataboica (Val-Alain, Quebec City); and Mont Atoca (Ste-Melanie, Quebec City). Following the harvest, the berries were sent to our laboratory and stored at $-20^{\circ} \mathrm{C}$ prior to processing. The berries were prepared for analysis by surface-sterilizing $(3 \mathrm{~min}$ in a $5 \%$ sodium hypochlorite solution, $3 \mathrm{~min}$ in a $70 \%$ ethanol solution, and a final 3-min rinse in sterile distilled water). To compare the cultural and molecular methods, each fruit was individually labeled and then cut longitudinally in half through the stem and calyx; the first half was used for traditional fungal isolation and identification, and the second half was used for direct diagnosis using the multiplex method.

Fruit DNA extraction. One half of each fruit was lyophilized and $50 \mathrm{mg}$ of berry $(\approx 400 \mathrm{mg}$ of fresh fruit) was pulverized prior to DNA extraction using the methodology described previously for pure fungal strains. The cetyltrimethylamonium bromide (CTAB) method described previously (Doyle and Doyle 1987) was adapted for DNA extraction as follows: the pulverized tissue was incubated in $1 \mathrm{ml}$ of CTAB Tris-5X Buffer $(500 \mathrm{mM}$ TrisHCl at $\mathrm{pH} 8,1.4 \mathrm{M} \mathrm{NaCl}$, $50 \mathrm{mM}$ EDTA, $1 \%$ polyvinyl-pyrrolidone); and DNA was precipitated by adding $700 \mu \mathrm{l}$ of chloroform:isoamylalcohol (24:1) and extracted using the E.Z.N.A. plant DNA kit (Omega Bio-tek) according to the manufacturer's protocol for lyophilized material. Primers used for amplification of cranberry are listed in Table 3.

Fungal isolation from fruit and isolate DNA extraction. The second half of each surface-disinfected fruit was placed with its cut surface down onto PDA (39 g/liter) amended with penicillin $(50 \mathrm{mg} /$ liter) to inhibit bacteria. Plates were incubated at room temperature $\left(25^{\circ} \mathrm{C}\right)$ and examined at least once per week until no additional fungi were observed. Fungi were transferred as soon as the presence of mycelial growth was detected on the culture medium containing the fruit samples. The mycelium fragments were transferred onto a clean PDA plate to obtain pure fungal cultures. The individual fungal cultures were kept at room temperature until DNA extraction, which was performed with E.Z.N.A. plant DNA Kit (Omega Bio-tek), as described for pure fungal strains.

Sequencing and identification of fungal isolates. The universal fungal-specific primer pair, ITS5 (White et al. 1990) and LR5 (Vilgalys and Hester 1990), was used to obtain ITS-LSU sequences for each of the fungi isolated from the fruit samples. The PCR and sequencing procedures were performed as previously described for pure strains. Isolate identification was performed by using the BLAST method (Ross et al. 2008). For each isolated strain, the ITS-LSU region was used as a query sequence in the standard nucleotide BLAST for NCBI fungal organism database only (Altschul 1997). The best BLAST hits that provided alignments with all or a part of the target DNA sequences with an $E$ value $<10^{-4}$ were considered identifications of positive species. Furthermore, a comparison with the 12 reference sequence alignments at our disposal was performed.

Evaluation of the multiplex PCR-based diagnostic assay. The capacity to detect the targeted CFR fungi directly from inoculated fruit was validated using DNA extracted directly from fruit experimentally inoculated with known fungi. The controlled inoculation was performed using surface-sterilized healthy fruit cut into halves and placed in a Petri dish with fungal isolates. These cultures were kept 
at room temperature for 2 weeks until fruits were visually colonized. The DNA extraction was performed as described.

\section{Results}

Development and optimization of multiplex PCR. Specific primer design and uniplex validation. Based on the ITS-LSU sequence analysis, 10 forward primers and seven reverse primers were designed to detect the target CFR fungi (Table 3) in a multiplex PCR (Figs. 1 and 2). Four species-specific forward primers were designed to work with a single universal reverse primer to amplify unique sequences from Phyl. elongata, M. oxycocci, P. vaccinii, and Phyl. vaccinii. The amplicons ranged from 368 to 518 bp. For C. empetri, F. putrefaciens, and Phys. vaccinii, a specific primer pair was designed for each species, and the resulting amplicons ranged in size from 500 to $700 \mathrm{bp}$.

Because of the high similarity of the ITS-LSU regions among A. cytisporea, A. lycopodina, and S. geniculata (Phacidiaceae), as well as between $C$. fioriniae and $C$. fructivorum (Glomerellaceae), a different approach was necessary. Therefore, family-specific primer pairs were developed to identify fungi as Phacidiaceae and Glomerellaceae

Table 3. List of specific primers used in this study for the identification of 12 fungi and cranberry

\begin{tabular}{|c|c|c|c|c|}
\hline Specie target & Region & Forward and reverse sequence & $\mathbf{b p}^{\mathbf{y}}$ & $\begin{array}{l}\text { Reaction } \\
\text { group }\end{array}$ \\
\hline Phomopsis vaccinii & ITS-LSU & $\begin{array}{l}\text { Fw: ACACTGAAACTCTGAGAATAAAACAT; } \\
\text { Rv: CCTACCTGATCCGAGGTCAAC }\end{array}$ & 368 & A \\
\hline Phacidiaceae & ITS-LSU & Fw: GTCGCAAGACAACCGGC; Rv: CCTACCTGATCCGAGGTCAAC & 400 & A \\
\hline Monilinia oxycocci & ITS-LSU & Fw: CCACAGGGGCAGAACCTCTC; Rv: CCTACCTGATCCGAGGTCAAC & 458 & A \\
\hline Phyllosticta vaccinii & ITS-LSU & Fw: CAGCACCCCTTGTGTACC; Rv: CCTACCTGATCCGAGGTCAAC & 518 & A \\
\hline Glomerellaceae & ITS-LSU & Fw: ACGGGTCGCTGATGACCATT; Rv: CACAGGCCAGCATCAGCTTG & 159 & B \\
\hline Physalospora vaccinii & ITS-LSU & Fw: AGCTCCCTTCGGGGAGTGTT; Rv: GCTTCACCCTACGCGAGCAT & 293 & B \\
\hline Phyllosticta elongata & ITS-LSU & Fw: CCGCCAGTACACAAAACTCC; Rv: CCTACCTGATCCGAGGTCAAC & 410 & B \\
\hline Fusicoccum putrefaciens & ITS-LSU & Fw: CGCTTGCGTCCATGAAGT; Rv: GCAAAGAGCCTAGATGAGCAAC & 500 & B \\
\hline Coleophoma empetri & ITS-LSU & Fw: GTATGTGATCGGTTGCTTGC; Rv: TTTCCTCTGGCTTCACCCTA & 700 & B \\
\hline Allantophomopsis cytisporea & TEF- $1 \alpha$ & Fw: CTCAGCGGCTTCCTGAATA; Rv: GTGGGGGTATAGACTCGCTTC & 174 & $\mathrm{C}$ \\
\hline Allantophomopsis lycopodina & TEF- $1 \alpha$ & Fw: CTCAGCGGCTTCCTGAATA; Rv: GTGGGGGTATAGACTCGCTTC & 734 & $\mathrm{C}$ \\
\hline Strasseria geniculata & TEF- $1 \alpha$ & Fw: CTCAGCGGCTTCCTGAATA; Rv: GTGGGGGTATAGACTCGCTTC & $-\mathrm{z}$ & $\mathrm{C}$ \\
\hline Colletotrichum fiorinae & ITS-LSU & Fw: GGACAAAAGCTTCAGGAACG; Rv: TTTCCTCTGGCTTCACCCTA & 325 & $\mathrm{D}$ \\
\hline Vaccinium macrocarpon & ITS-LSU & Fw: TTAGGCTGAAGGCACGTCTG; Rv: TTAAACGGCACTCCAGGGTC & 258 & - \\
\hline
\end{tabular}

$\mathrm{y} \mathrm{bp}=$ Base pair size of the fungal DNA fragment amplified.

${ }^{\mathrm{z}}$ Dashes indicate no DNA amplification.

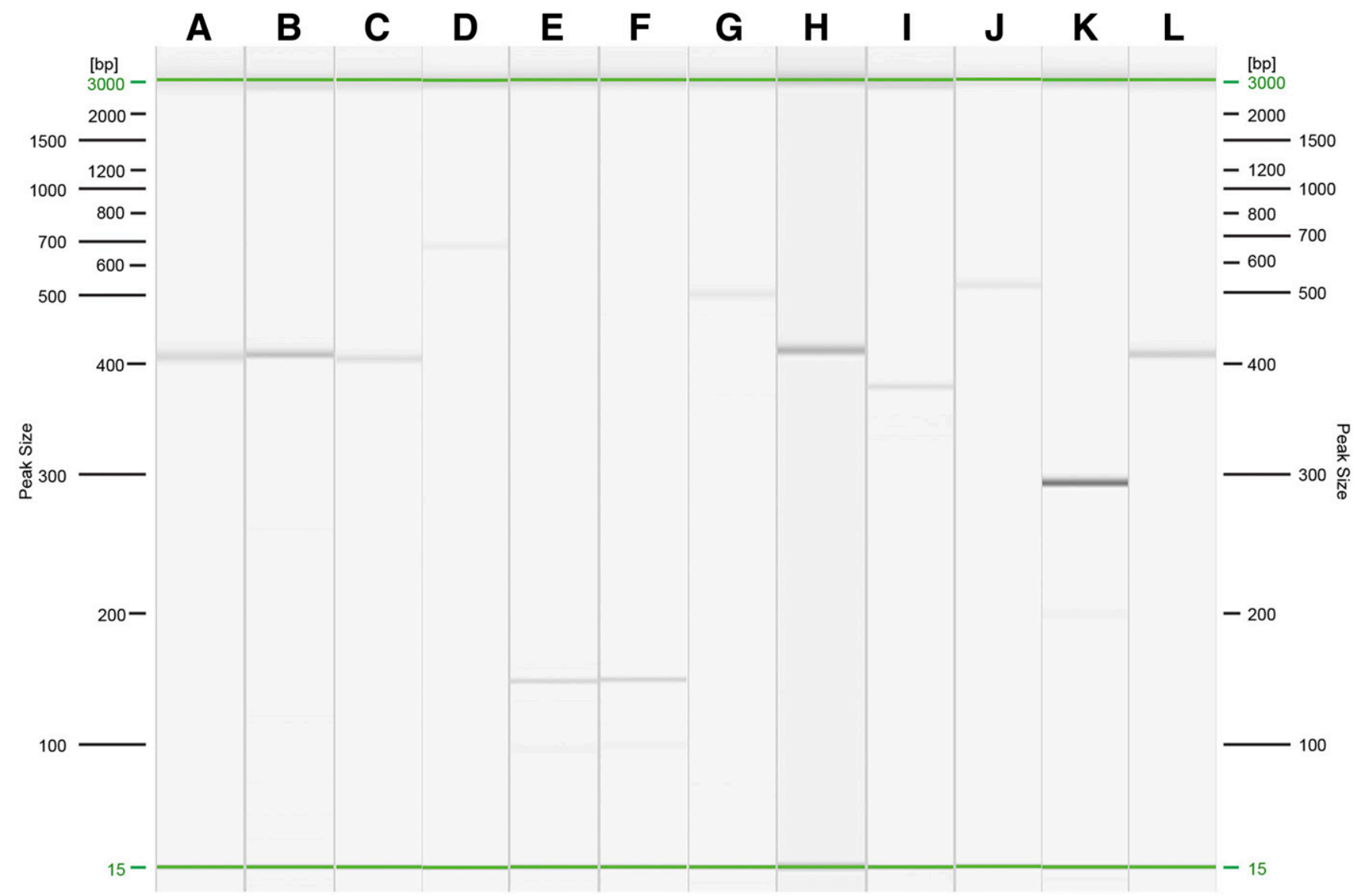

Fig. 1. Gel image showing the band sizes for each species. A, Allantophomopsis cytisporea; B, Allantophomopsis lycopodina; C, Phyllosticta elongata; D, Coleophoma empetri; E, Colletotrichum fiorinae; F, Colletotrichum fructivorum; G, Fusicoccum putrefaciens; H, Monilinia oxycocci; I, Phomopsis vaccinii; J, Phyllosticta vaccinii; K, Physalospora vaccinii; and L, Strasseria geniculata. 
with amplicon sizes of $400 \mathrm{bp}$ and $159 \mathrm{bp}$, respectively. It was possible to develop a primer pair in the TEF- $1 \alpha$ region to differentiate the three Phacidiaceae species. The amplicon sizes for A. cytisporea and A. lycopodina were, respectively, 174 and $734 \mathrm{bp}$; however, the absence of an amplicon confirmed the diagnosis of $S$. geniculata in case of positive amplification with family-specific primers (Fig. 2). Similarly, for the Glomerellaceae-specific primers, one primer was designed to differentiate $C$. fioriniae (one amplicon at $325 \mathrm{bp}$ ) from $C$. fructivorum (no amplicon) (Fig. 3).

Multiplex PCR development and optimization. For optimal resolution of the molecular assay, the multiplex was divided into four reactions (A to D): two main reactions (A to B) involved amplifying seven species and two families, and two complementary reactions (C to D) were needed only if primers of Phacidiaceae and Glomerellaceae generated amplifications. The first group (reaction A) was designed to amplify A. cytisporea, A. lycopodina, S. geniculata, M. oxycocci, $P$. vaccinii, and Phyl. vaccinii. The second group (reaction B) amplified Phyl. elongata, C. empetri, C. fiorinae, C. fructivorum, $F$. putrefaciens, and Phys. vaccinii. In the case of a positive reaction with members of Phacidiaceae, a third group (reaction C) was used. Finally, the fourth group (reaction D) allowed discrimination of the Colletotrichum species when a Glomerellaceae member was detected in reaction $\mathrm{B}$. The complete list of specific primers is shown in Table 3.

PCR efficiency was similar between the uniplex and multiplex reactions. However, multiplex optimization was performed by adjusting the concentration balance for each primer pair during PCR (Table 4). For each reaction, $1 \mu \mathrm{l}$ of the forward primer mixtures and
$1 \mu l$ of the reverse primer mixtures were added, and the forward/ reverse primer concentrations were set as shown in Table 4 (according to the optimum concentration obtained in the uniplex gradient). The only exception was $P$. vaccinii, which had forward and reverse primer concentrations of $0.05 \mu \mathrm{M}$ and $0.002 \mu \mathrm{M}$, respectively.

Sensitivity of specific primers. The sensitivity test of the primer pairs performed with serial DNA dilutions for each CFR fungus determined that the threshold of DNA detection was $10^{-2} \mathrm{pg}$, depending on the primer pair (Table 4). Therefore, detection sensitivity was extremely high for all species tested in this study.

Validation of the multiplex on fruit samples. Fungal isolation from fruit and identification. Our isolation procedure yielded $300 \mathrm{pu}-$ re fungal isolates from the 117 cranberry fruit. Many of them were represented by fungi that were not pathogenic to cranberry, such as Trichoderma spp, Mucor sp., and Penicillium sp., based on a BLAST search with ITS-LSU sequences. Altogether, 23 isolates were positively identified as one of the 12 fungi causing CFR. Of the 12 target fungi, the most commonly found were Phys. vaccinii (53\%), followed by C. empetri (17\%) and the Phacidiaceae family (12\%; all S. geniculata). Phyl. elongata, $P$. vaccinii, and $C$. fructivorum were also found in proportions of $6 \%$ each (Fig. 4). A. cytisporea, A. lycopodina, $C$. fiorinae, $F$. putrefaciens, $M$. oxycocci, and Phyl. vaccinii were not found.

Comparison between isolate identification and multiplex diagnosis. Figure 5 shows an example of a PCR analysis performed for three fungi that were detected by amplifications using the molecular tools developed during this study of cranberry DNA. The

\section{Reaction A}

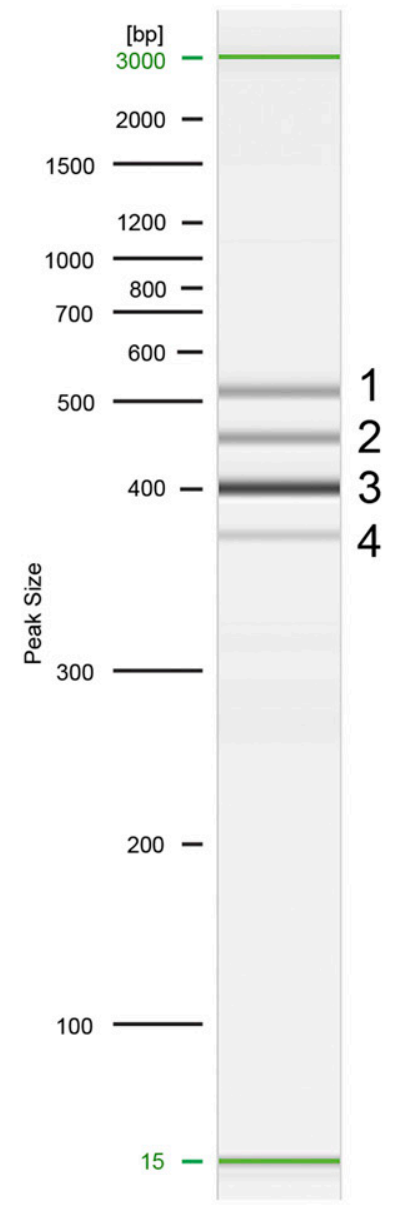

Reaction B

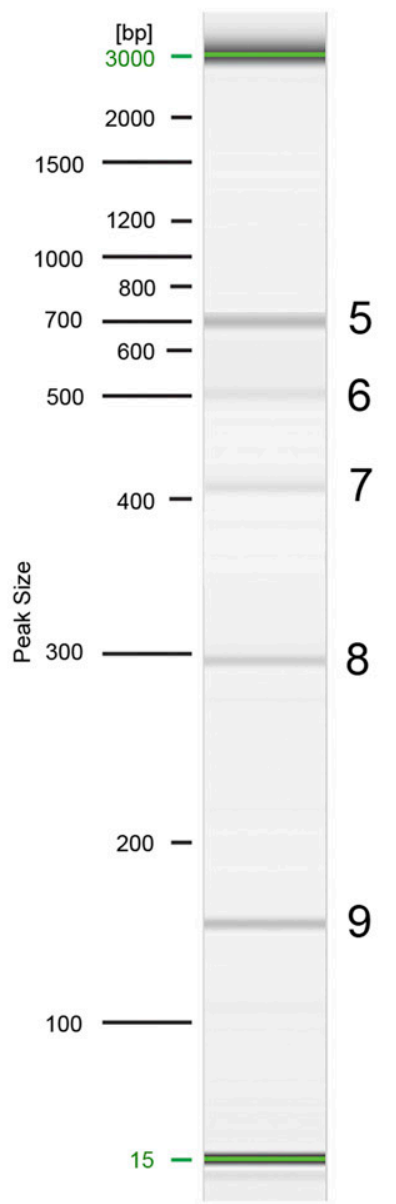

Reaction C

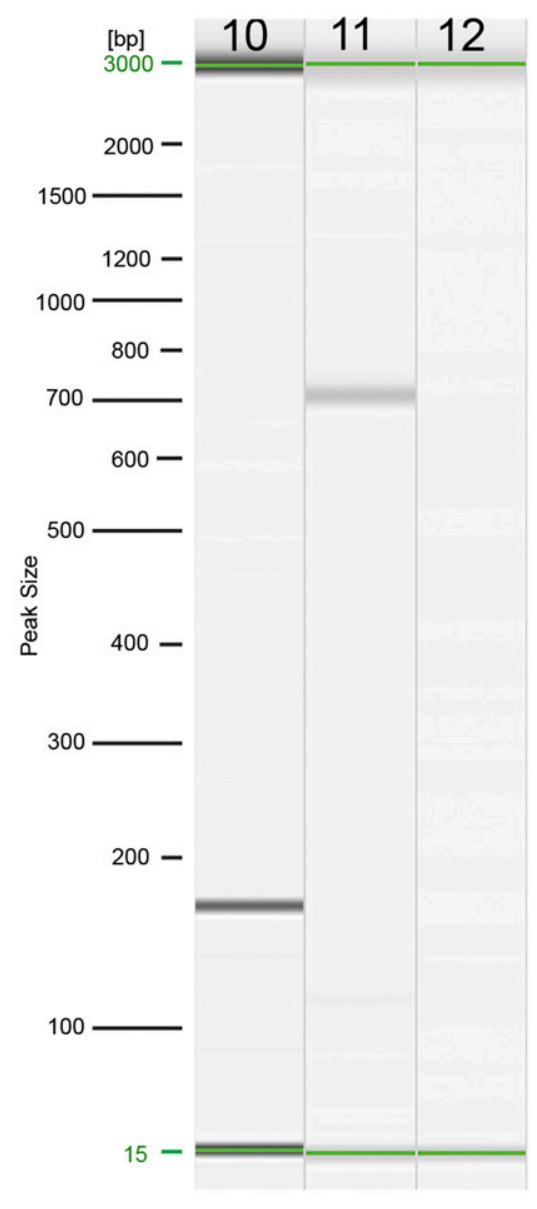

Fig. 2. Gel image of the three reactions. A, Amplifying (1) Phyllosticta vaccinii (518 bp); (2) Monilinia oxycocci (458 bp); (3) Phacidiaceae family (396 to 416 bp); and (4) Phomopsis vaccinii (368 bp). B, Amplifying (5) Coleophoma empetri (700 bp); (6) Fusicoccum putrefaciens (500 bp); (7) Phyllosticta elongata (410 bp); (8) Physalospora vaccinii (293 bp); and (9) Glomerellaceae family (159 bp). C, The difference in amplifications between members of the Phacidiaceae using the TEF-1 $\alpha$-specific primers (10) Allantophomopsis cytisporea (174 bp), (11) Allantophomopsis lycopodina (734 bp), and (12) Strasseria geniculata (no amplification). 
diagnosis was confirmed by an analysis of ITS-LSU sequences that are publicly available. For all 23 fungi identified for cranberry fruit, we found consistent matches between the multiplex PCR-based and the BLAST-based diagnoses. All pathogenic fungi identified by the multiplex PCR were isolated from the fruit in the same manner.

\section{Discussion}

CFR is a disease complex involving several fungal pathogens that can induce rot symptoms (Oudemans et al. 1998; Polashock et al. 2017). A proper diagnosis based on traditional isolation procedures is necessary, but it can be both complex and time-consuming. Therefore, a more modern approach, as described here, should streamline sample processing significantly. In this study, 12 fungal species commonly associated with CFR were successfully identified using a multiplex PCR approach with a unique series of primers divided in four groups. This new diagnostic tool offers a more precise and rapid approach to identifying the pathogen complex responsible for CFR.

The design process included sequences obtained from fungal isolates using the generic ITS5-LR5 primers, followed by alignments performed using sequences available in the NCBI database. By using the large NCBI database, we were able to confidently identify the target regions for discriminating each species. However, to properly design a multiplex procedure, we had to overcome two main

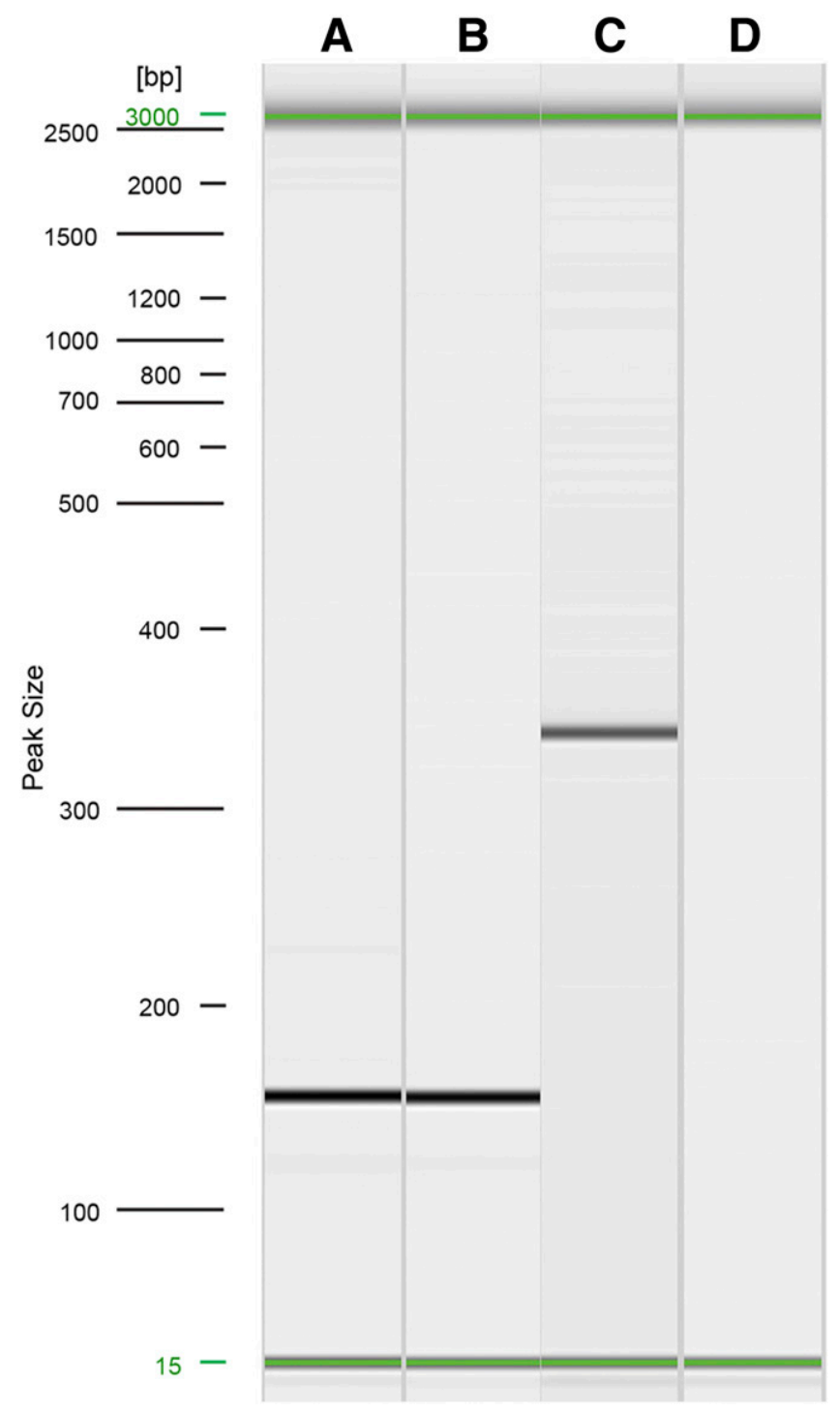

Fig. 3. Gel image showing reaction $D$, discriminating the two Colletotrichum complexes. A and B, Glomerellaceae family-specific primer on A, Colletotrichum fiorinae and $\mathbf{B}$, Colletotrichum fructivorum. $\mathbf{C}$ and $\mathbf{D}$, Discriminating primer on $\mathbf{C}$. fiorinae (C, one band at $325 \mathrm{bp}$ ) and $\mathrm{C}$. fructivorum (D, no amplification). difficulties. The first was related to a lack of species resolution of the ITS-LSU region among the target fungi. Using this sequence, it was possible to differentiate seven of the 12 species and two families that contained the remaining five species. Three species belonging to the Phacidiaceae and two in the Glomerellaceae family could not be clearly discriminated using ITS-LSU-based primers. Among Phacidiaceae, there were only two SNPs, and they could not be exploited to design primers for differentiating the species. In such cases, alternate molecular markers (TEF) have been shown to be useful (Raja et al. 2017). Using the TEF sequence, we designed primers to differentiate the three species within Phacidiaceae in a separate reaction when the ITS-LSU reaction had confirmed the presence of a member of this family. In the present study, only $S$. geniculata was detected among field-collected samples.

It was equally problematic to identify the two species of Glomerellaceae. As a first approach, we chose to target the two Colletotrichum spp. together because $C$. fioriniae and $C$. fructivorum belong to two large species complexes (Adaskaveg and Hartin 1997; Damm et al. 2012; Doyle et al. 2013; Penet et al. 2017; Weir et al. 2012) in which more than two species may share $V$. macrocarpon as a host. As a result, we first identified a generic primer set to amplify isolates belonging to the genus Colletrotrichum. Subsequently, we designed a primer pair to differentiate isolates in the complex containing $C$. fioriniae from the one containing $C$. fructivorum that amplifies a single 325-bp fragment from the $C$. fioriniae group. This primer pair may provide unclear results if different strains within each complex are amplified; therefore, it may be more practical to simply limit the diagnosis to a Colletotrichum species. Considering that both

Table 4. Sensitivity of the multiplex reaction showing the minimum primer and DNA concentrations required for amplification of the 12 fungi causing cranberry fruit rot

\begin{tabular}{lcc}
\hline Species & $\begin{array}{c}\text { Minimum primer } \\
\text { concentration }(\boldsymbol{\mu M})\end{array}$ & $\begin{array}{c}\text { Minimum DNA } \\
\text { concentration } \\
(\mathbf{p g} / \boldsymbol{\mu l})\end{array}$ \\
\hline Phomopsis vaccinii & 0.05 & 0.25 \\
Coleophoma empetri & 0.02 & 0.25 \\
Physalospora vaccinii & 0.01 & 25 \\
Phyllosticta elongata & 0.005 & 0.025 \\
Colletotrichum fiorinae & 0.005 & 2.5 \\
Colletotrichum fructivorum & 0.005 & 2.5 \\
Fusicoccum putrefaciens & 0.005 & 0.25 \\
Monilinia oxycocci & 0.005 & 0.025 \\
Allantophomopsis cytisporea & 0.002 & 0.025 \\
Allantophomopsis lycopodina & 0.002 & 0.025 \\
Strasseria geniculata & 0.002 & 0.025 \\
Phyllosticta vaccinii & 0.001 & 0.0025 \\
\hline
\end{tabular}

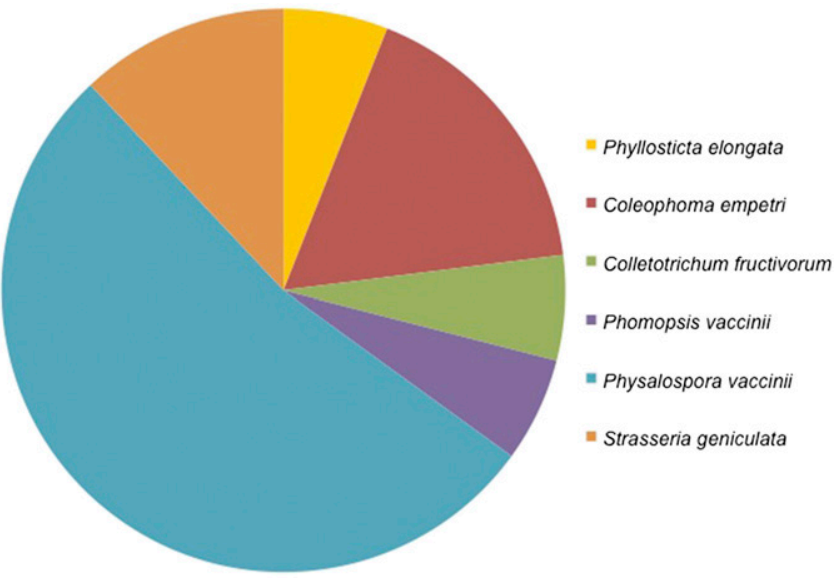

Fig. 4. Proportion of fungal pathogens causing cranberry fruit rot identified from 117 cranberry fruit based on a multiplex PCR assay and standard isolation techniques. 
Colletotrichum species cause similar symptoms and the same bitter rot, it may be legitimate to suggest that the diagnosis of a Colletotrichum spp. would involve a common fungicide treatment.

A second difficulty was that many of the species within the CFR complex are not host-specific and may be found on diverse hosts. The geographic origins and host specificity are two important factors resulting in the development of different strains within a genus or species, so it is likely that not all strains belonging to a species complex are pathogenic on cranberry (Raj 1983; Vilka and Volkova 2015). However, C. fructicola, for example, was identified as a new species (Doyle et al. 2013) from within the large species complex $C$. gloeosporioides. Isolates of this new species were consistently found to be associated with rotted cranberry fruit throughout North America; however, a small number of related species were occasionally detected on cranberry. In contrast, a second species, C. fioriniae, was identified as a member of the broad host range C. acutatum complex (Damm et al. 2012). C. fioriniae is also associated with rotted cranberry fruit and a number of other fruit crops. Given the history of the cranberry industry, bulk planting material was freely traded among growers from different regions, including all North American locations (Oudemans et al. 1998). Therefore, most pathogens associated with cranberry were also likely shared among regions. Not surprisingly, Polashock (2009) found strong identity within the major CFR species from different regions in North America, thus supporting the idea that these fungi share a common origin. However, we had to consider that some ITS-LSU sequences obtained from the isolates undergoing study were not typical of strains found on cranberry. To mitigate this possibility, our primers were designed following a comparison between the alignments performed with our 12 reference fungi and the sequences of the NCBI database and the sequences of the fungal pathogens isolated directly from cranberry fruit. This process will be repeated as we expand the number of fungi isolated on cranberry to ensure that the molecular assay is highly specific for cranberry pathogens.

The multiplex PCR method described here was subject to several steps of optimization. By using two initial reactions, A and B, the test resulted in the identification of seven species and two families (containing five species). All the species from reaction A share the same reverse primer (they have a unique forward primers), which would interfere with the primers in reaction B. Because these primers target distinct amplifications from the same genomic region, it was necessary to split the multiplex into two reactions as described, and this required optimization of the primer concentration. For instance, $P$. vaccinii, in reaction A, required an increase of its forward primer to $0.05 \mu \mathrm{M}$ to maximize its resolution. Regarding the DNA concentration, quantities as low as $0.01 \mathrm{pg}$ were sufficient to generate discriminant amplicons, which should be quite sufficient to detect minimal quantities, as reported for other pathogens such as Phytophthora sojae (ArsenaultLabrecque et al. 2018) or Phytophthora capsici (Smith et al. 2019).

The traditional isolation and culturing part of this study yielded $>300$ isolates that provided ample material to validate the efficiency of the multiplex procedure for detecting the targeted pathogenic fungi. Of 300 isolates, only 23 samples contained the fungi targeted by the multiplex. This was an interesting result, because if the conventional microscopic approach was used, then it would have been necessary to analyze the entire spectrum of 300 fungi. Based on the BLAST analysis, the majority of those 300 fungi were not listed as pathogenic toward cranberry (Polashock et al. 2017); therefore, an elaborate and comprehensive microscopic analysis would have required much more time than the multiplex method. The whole isolation process required more than 2 months, whereas the PCR diagnostic, including DNA extraction, required $\approx 6 \mathrm{~h}$. Interestingly, the PCR method revealed the same pathogenic fungi as those identified through the standard plating isolation technique and did not amplify false-negative results despite the numerous contaminants isolated from the fruit. However, our results showed that cranberry fruit, whether healthy or infected, harbor a diverse microbiome.

Based on the validation studies of field-collected fruit of the fall cultivars Stevens, Benlear, and Bergman, Phys. vaccinii was found to be the pathogen most commonly associated with CFR in our samples. This species is responsible for blotch rot in cranberry (Polashock et al. 2017), and it has been frequently reported in the literature as a pathogen of cranberry (Blodgett et al. 2002; Olatinwo et al. 2003; Polashock et al. 2009). Therefore, it is not surprising that it also represented the majority of the isolates of known pathogenic fungi identified in our study. C. empetri was second in abundance, and it is also commonly reported to cause ripe rot on fruit. Members of Phacidiaceae and S. geniculata in particular were found in $12 \%$ of our positive samples. As a group, they are responsible for black rot (Carris 1990), which is an emerging problem for cranberry growers' fresh fruit, particularly in the Northeast. These are capable of infecting stems, flowers, and leaves, although little is known about their epidemiology. Given the accuracy and sensitivity of the multiplex PCR tool described here, it could be used to improve the understanding of life cycles and biology of the black rot fungi and ultimately optimize ways to manage the disease. Because it is difficult to detect these fungi before symptom development, molecular detection may be an essential tool for this type of research.

Regarding the field-collected fruit samples, only six of the 12 target fungi were detected among the 117 fruit samples tested. Many factors can explain this result, including the fact that all fruit were sampled during the late fall period. For instance, the absence of $M$. oxycocci is likely attributable to its ability to infect the flower's stigma during the early stage of the culture, which would make it easier to detect in the spring (McManus et al. 1999; Sanderson and

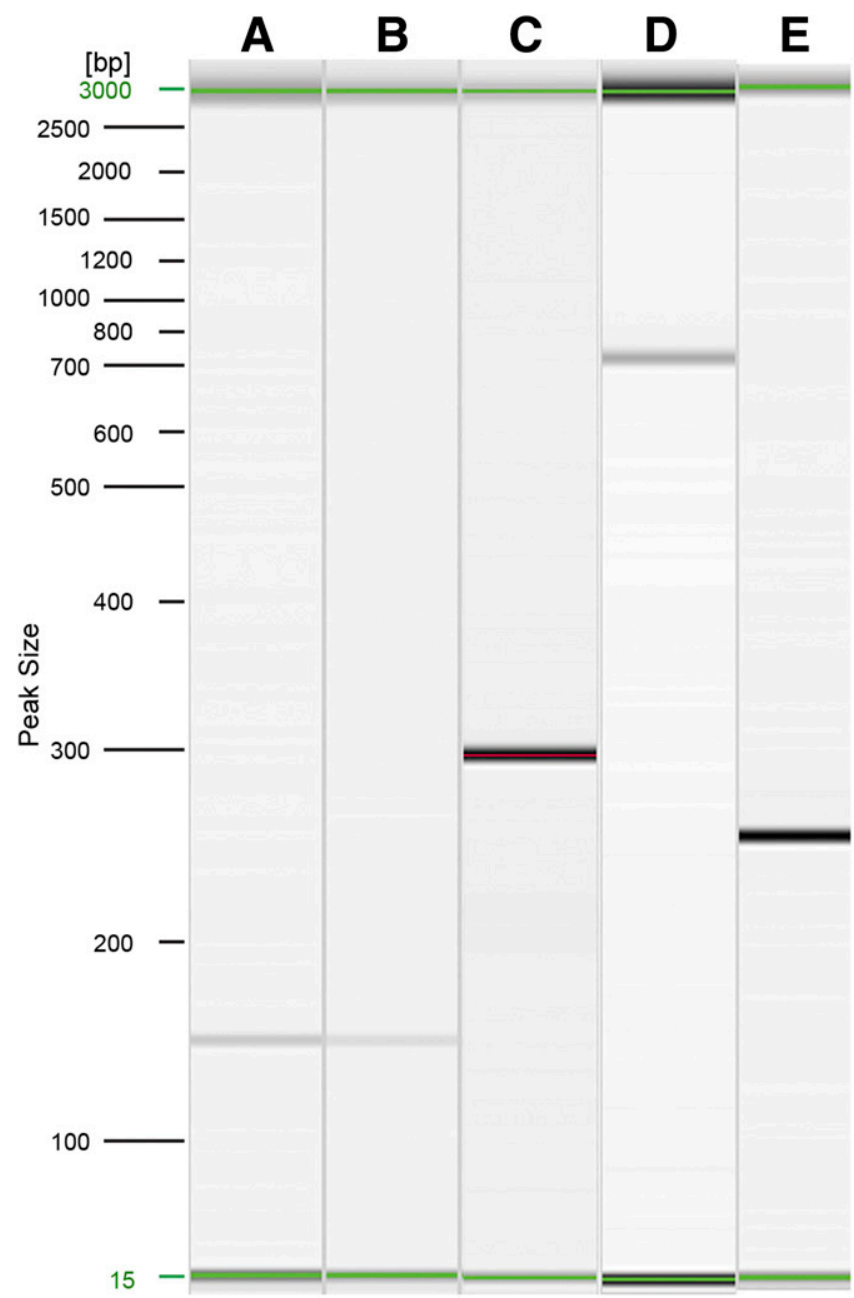

Fig. 5. Gel image of the multiplex performed on four cranberry fruit. The amplicons show the presence of A and B, Colletotrichum sp. (159 bp), C, Physalospora vaccinii (293 bp), and D, Coleophoma empetri (700 bp) The amplification in $\mathbf{E}$, represents the control with universal primers targeting cranberry DNA (258 bp). 
Jeffers 1992). Geographical considerations equally influence the representation of fungi. Our sampling was limited to cranberry beds from Québec and Nova Scotia. Therefore, it is likely that fungal populations differ in British Columbia, Washington, and Oregon or in other important cranberry production areas such as Wisconsin and Massachusetts, where climatic conditions differ. It will be quite interesting to implement the use of this molecular assay at different times of the season and throughout the areas of cranberry production in North America to obtain a rapid and reliable assessment of the presence and progression of fungi involved in CFR.

In conclusion, the results described in this study demonstrate a molecular assay for the diagnosis of the 12 primary fungi responsible for CFR. This assay eliminates the long and complex steps of fungal isolation and identification and offers precise, reliable, and sensitive detection of fungi directly from fruit tissues. It should have many applications in the management of CFR, especially in the context of improving the understanding of disease epidemiology. In addition, it will offer cranberry growers a useful tool for tailoring the application of fungicide treatments for the control of disease.

\section{Acknowledgments}

We gratefully acknowledge Jean-Pierre Deland at Ocean Spray for the generous donation of several fruit samples. Particular thanks are extended to Caroline Labbé for her contribution to this study.

\section{Literature Cited}

Adaskaveg, J. E., and Hartin, R. J. 1997. Characterization of Colletotrichum acutatum isolates causing anthracnose of almond and peach in California. Phytopathology 87:979-987.

Altschul, S. 1997. Gapped BLAST and PSI-BLAST: a new generation of protein database search programs. Nucleic Acids Res. 25:3389-3402.

Arsenault-Labrecque, G., Sonah, H., Lebreton, A., Labbé, C., Marchand, G., Xue, A., Belzile, F., Knaus, B. J., Grünwald, N. J., and Bélanger, R. R. 2018. Stable predictive markers for Phytophthora sojae avirulence genes that impair infection of soybean uncovered by whole genome sequencing of 31 isolates. BMC Biol. 16:80.

Aslam, S., Tahir, A., Aslam, M. F., Alam, M. W., Shedayi, A. A., and Sadia, S. 2017. Recent advances in molecular techniques for the identification of phytopathogenic fungi - a mini review. J. Plant Interact. 12:493-504.

Blodgett, A. B., Caldwell, R. W., and McManus, P. S. 2002. Effects of calcium salts on the cranberry fruit rot disease complex. Plant Dis. 86:747-752.

Bruns, T. D., and Shefferson, R. P. 2004. Evolutionary studies of ectomycorrhizal fungi: recent advances and future directions. Can. J. Bot. 82:1122-1132.

Burbank, L. P., and Ortega, B. C. 2018. Novel amplification targets for rapid detection and differentiation of Xylella fastidiosa subspecies fastidiosa and multiplex in plant and insect tissues. J. Microbiol. Methods 155:8-18.

Carbone, I., and Kohn, L. M. 1999. A method for designing primer sets for speciation studies in filamentous ascomycetes. Mycologia 91:553-556.

Carris, L. M. 1990. Cranberry black rot fungi: Allantophomopsis cytisporea and Allantophomopsis lycopodina. Can. J. Bot. 68:2283-2291.

Damm, U., Cannon, P. F., Woudenberg, J. H. C., and Crous, P. W. 2012. The Colletotrichum acutatum species complex. Stud. Mycol. 73:37-113.

Doyle, J. J., and Doyle, J. L. 1987. A rapid DNA isolation procedure for small quantities of fresh leaf tissue. Phytochem. Bull. 19:11-15.

Doyle, V.P., Oudemans, P.V., Rehner, S.A., Litt, A., 2013. Habitat and host indicate lineage identity in Colletotrichum gloeosporioides s.l. from wild and agricultural landscapes in North America. PLoS ONE 8:e62394.

Gallardo, R. K., Zhang, Q., Dossett, M., Polashock, J. J., Rodriguez-Saona, C., Vorsa, N., Edger, P. P., Ashrafi, H., Babiker, E., Finn, C. E., and Iorizzo, M. 2018. Breeding trait priorities of the blueberry industry in the United States and Canada. HortScience 53:1021-1028.

Kostov, K., Verstappen, E. C. P., Bergervoet, J. H. W., de Weerdt, M., Schoen, C. D., Slavov, S., and Bonants, P. J. M. 2016. Multiplex detection and identification of Phytophthora spp. using target-specific primer extension and Luminex xTAG technology. Plant Pathol. 65:1008-1021.

Lang, J. M., Hamilton, J. P., Diaz, M. G. Q., Van Sluys, M. A., Burgos, M. R. G., Vera Cruz, C.M., Buell, C.R., Tisserat, N.A., and Leach, J.E. 2010. Genomicsbased diagnostic marker development for Xanthomonas oryzae pv. oryzae and X. oryzae pv. oryzicola. Plant Dis. 94:311-319.

Marin-Felix, Y., Groenewald, J. Z., Cai, L., Chen, Q., Marincowitz, S., Barnes, I., Bensch, K., Braun, U., Camporesi, E., Damm, U., de Beer, Z. W., Dissanayake, A., Edwards, J., Giraldo, A., Hernández-Restrepo, M., Hyde, K. D., Jayawardena, R. S., Lombard, L., Luangsa-ard, J., McTaggart, A. R., Rossman, A. Y., Sandoval-Denis, M., Shen, M., Shivas, R. G., Tan, Y. P., van der Linde, E. J., Wingfield, M. J., Wood, A. R., Zhang, J. Q., Zhang, Y., and Crous, P. W. 2017. Genera of phytopathogenic fungi: GOPHY 1. Stud. Mycol. 86:99-216.

Marin-Felix, Y., Hernández-Restrepo, M., Wingfield, M. J., Akulov, A., Carnegie, A. J., Cheewangkoon, R., Gramaje, D., Groenewald, J. Z., Guarnaccia, V.,
Halleen, F., Lombard, L., Luangsa-ard, J., Marincowitz, S., Moslemi, A., Mostert, L., Quaedvlieg, W., Schumacher, R. K., Spies, C. F. J., Thangavel, R., Taylor, P. W. J., Wilson, A. M., Wingfield, B. D., Wood, A. R., and Crous, P. W. 2019. Genera of phytopathogenic fungi: GOPHY 2. Stud. Mycol. 92:47-133.

Martinelli, F., Scalenghe, R., Davino, S., Panno, S., Scuderi, G., Ruisi, P., Villa, P., Stroppiana, D., Boschetti, M., Goulart, L. R., Davis, C. E., and Dandekar, A. M. 2015. Advanced methods of plant disease detection. A review. Agron. Sustain. Dev. 35:1-25.

McManus, P. S., Best, V. M., and Voland, R. P. 1999. Infection of cranberry flowers by Monilinia oxycocci and evaluation of cultivars for resistance to cottonball. Phytopathology 89:1127-1130.

McManus, P. S., Caldwell, R. W., Voland, R. P., Best, V. M., and Clayton, M. K. 2003. Evaluation of sampling strategies for determining incidence of cranberry fruit rot and fruit rot fungi. Plant Dis. 87:585-590.

Michalecka, M., Bryk, H., Poniatowska, A., and Puławska, J. 2016. Identification of Neofabraea species causing bull's eye rot of apple in Poland and their direct detection in apple fruit using multiplex PCR. Plant Pathol. 65: 643-654.

Mukezangango, J. 2017. Statistical overview of the Canadian fruit industry 2016. Agriculture and Agri-Food Canada No. 12689E. Government of Canada, Ottawa, $34 \mathrm{p}$.

O’Donnell, K., Kistler, H. C., Cigelnik, E., and Ploetz, R. C. 1998. Multiple evolutionary origins of the fungus causing Panama disease of banana: Concordant evidence from nuclear and mitochondrial gene genealogies. Proc. Natl. Acad. Sci. 95:2044-2049.

Olatinwo, R. O., Hanson, E. J., and Schilder, A. M. C. 2003. A first assessment of the cranberry fruit rot complex in Michigan. Plant Dis. 87:550-556.

Oudemans, P. V., Caruso, F. L., and Stretch, A. W. 1998. Cranberry fruit rot in the Northeast: a complex disease. Plant Dis. 82:1176-1184.

Penet, L., Briand, S., Petro, D., Bussière, F., and Guyader, S. 2017. Data on microsatellite markers in Colletotrichum gloeosporioides s.l., polymorphism levels and diversity range. Data Brief 12:644-648.

Polashock, J. J., Caruso, F. L., Averill, A. L., and Schilder, A. 2017. Compendium of Blueberry, Cranberry, and Lingonberry Diseases and Pests, 2nd Ed. APS Press, St. Paul, MN, 231 pp.

Polashock, J. J., Caruso, F. L., Oudemans, P. V., McManus, P. S., and Crouch, J. A. 2009. The North American cranberry fruit rot fungal community: a systematic overview using morphological and phylogenetic affinities. Plant Pathol. 58:1116-1127.

Raj, T. N. 1983. Genera coelomycetum. XXI. Strasseria and two new anamorphgenera, Apostrasseria and Nothostrasseria. Can. J. Bot. 61:1-30.

Raja, H. A., Miller, A. N., Pearce, C. J., and Oberlies, N. H. 2017. Fungal identification using molecular tools: a primer for the natural products research community. J. Nat. Prod. 80:756-770.

Reich, J. D., Alexander, T. W., and Chatterton, S. 2016. A multiplex PCR assay for the detection and quantification of Sclerotinia sclerotiorum and Botrytis cinerea. Lett. Appl. Microbiol. 62:379-385.

Robideau, G. P., Caruso, F. L., Oudemans, P. V., McManus, P. S., Renaud, M. A., Auclair, M. E., Bilodeau, G. J., Yee, D., Désaulniers, N. L., DeVerna, J. W., and Lévesque, C. A. 2008. Detection of cranberry fruit rot fungi using DNA array hybridization. Can. J. Plant Pathol. 30:226-240.

Ross, H. A., Murugan, S., and Sibon Li, W. L. 2008. Testing the reliability of genetic methods of species identification via simulation. Syst. Biol. 57:216-230.

Sanderson, P. G., and Jeffers, S. N. 1992. Cranberry cottonball: dispersal periods of primary and secondary inocula of Monilinia oxycocci, host susceptibility, and disease development. Phytopathology 82:384-392.

Schoch, C. L., Seifert, K. A., Huhndorf, S., Robert, V., Spouge, J. L., André Levesque, C., Chen, W., and Fungal Barcoding Consortium. 2012. Nuclear ribosomal internal transcribed spacer (ITS) region as a universal DNA barcode marker for Fungi. Proc. Natl. Acad. Sci. 109:6241-6246.

Silva, A. O., Savi, D. C., Gomes, F. B., Gos, F. M. W. R., Silva, G. J., and Glienke, C. 2017. Identification of Colletotrichum species associated with postbloom fruit drop in Brazil through GAPDH sequencing analysis and multiplex PCR. Eur. J. Plant Pathol. 147:731-748.

Smith, B. A. M., Eudoxie, G., and Saravanakumar, D. 2019. Identification of Phytophthora capsici causing collar rot in hot peppers in Trinidad. Can. J. Plant Pathol. 41:129-134.

Vilgalys, R., and Hester, M. 1990. Rapid genetic identification and mapping of enzymatically amplified ribosomal DNA from several Cryptococcus species. J. Bacteriol. 172:4238-4246.

Vilka, L., and Volkova, J. 2015. Morphological diversity of Phomopsis vaccinii isolates from cranberry (Vaccinium macrocarpon Ait.) in Latvia. Proc. Latv. Univ. Agric. 33:8-18.

Weir, B. S., Johnston, P. R., and Damm, U. 2012. The Colletotrichum gloeosporioides species complex. Stud. Mycol. 73:115-180.

Wells, L. D., and McManus, P. S. 2013. A photographic diagnostic guide for identification of the principal cranberry fruit rot pathogens. Plant Health Prog. 14:34.

White, T. J., Bruns, T. D., Lee, S. B., and Taylor, J. W. 1990. Amplification and direct sequencing of fungal ribosomal RNA genes for phylogenetics. Pages 315-322 in: PCR Protocols: Methods and Applications. M. A. Innis, D. H. Gelfand, J. L. Sninsky, and T. J. White, eds. Academic Press, San Diego, CA. 